Dr. Lines replies as follows:

The findings of Mesmacque-Caby, Farriaux, and Fontaine are contrary to those found by Swanson and myself which we have previously published. These differences may be due to a difference in definition of hyperphenylalaninaemia as suggested because we did not consider the absence of urinary metabolities necessary to define hyperphenylalaninaemia.

It is most important to emphasize that we no longer treat children for hyperphenylalaninaemia as pointed out in our original article. Thus, in the absence of treatment a deficiency state would not arise. Our article pointed out that we did no longer think treatment should be undertaken and certainly we would not propose a very strict phenylalanine restriction as our article suggests is necessary. If the data of Mesmacque-Caby, Farriaux, and Fontaine are correct and a low plasma phenylalanine can be maintained by mild diet, such a diet would not be likely to produce a deficiency state. However, as hyperphenylalaninaemia as defined by the above authors and ourselves has not been convincingly shown to cause mental retardation, such a diet appears to be unnecessary in either instance.

Also, as pointed out by numerous authors, hyperphenylalaninaemia is probably a heterogenous condition and in the light of this it is perhaps not surprising that some patients with it will require severe phenylalanine restrictions similar to the diet of phenylketonuria, while others would tolerate a higher amount of phenylalanine.

D. R. LINES

Department of Paediatrics, School of Medicine,

The University of Auckland, Auckland, N.Z.

\section{Head rotation and jugular vein blood flow}

Sir,

I read with interest the article 'Effect of head rotation on jugular vein blood flow' by G. H. Watson, p. 237, March 1974, Archives. Dr. Watson showed by angiographic technique that rotation of the head to one side causes occlusion of the ipsilateral internal jugular vein. This study confirms the clinical observation that during venepuncture of the internal jugular vein, ipsilateral rotation of the head stops the blood flow. It is important to realize this and when difficulty is encountered in withdrawing blood after a seemingly successful venepuncture of the internal jugular vein, one should first move the head to a midline position before poking around the neck with the needle.

VIVIAN E. SHIH

Massachusetts General Hospital, Boston, Mass. 02114, U.S.A.

\section{Congenital adrenal hyperplasia}

Sir,

We would like to comment upon three statements made by Dr. G. H. Newns in his annotation on congenital adrenal hyperplasia (Archives, 1974, 49, 1).

First, having pointed out that we had reported satisfactory growth in affected infants treated with prednisone trimethyl acetate (Bailey and Komrower, 1974), he rejects the treatment because of '. . . the known stunting effect of prednisone in early life . . . One of our reasons for presenting this paper was to show that infants could grow satsifactorily when placed on this treatment, which has considerable advantages in management and avoids loss of the drug due to vomiting or refusal to feed (Komrower and Longson, 1961).

Secondly, he states that the determination of urinary pregnanetriol is unnecessary. We have found this a very useful measurement in assessment of the control of these children.

The third and most important statement relates to the use of mineralocorticords. Dr. Newns says 'most salt losers can stop taking salt-retaining hormones at 4 to 5 years of age'. This is a dangerous statement. Children appear not to need salt-retaining hormones because they eat very large amounts of salt; some children take as much as $15 \mathrm{~g}$ a day. For this reason they suffer in two ways.

(1) This is a situation of electrolyte 'brinkmanship' and any inability on the child's part to take the usual large daily intake will precipitate a salt-losing crisis. In addition these children are generally not so fit and suffer from episodes of lassitude and nausea, with occasional vomiting, which disappear when mineralocorticord therapy is introduced.

(2) This constant loss of salt is a stressful situation and so stimulates the cholesterol-cortisol pathway. As a result, these children need more glucocorticoid therapy to maintain a satisfactory urine output of 17 -ketosteroids and pregnanetriol.

These two factors produce a more hazardous situation for these children and our experience in the past, coupled with a careful search of published reports, revealed a number of deaths which were almost certainly the result of salt rather than cortisol deficiency. This has not been seen since the introduction of fludrocortisone and it would be unfortunate if a retrograde step were taken which might lead to the recurrence of such deaths.

\section{G. M. KOMROWER and C. C. BAILEY Willink Biochemical Genetics Laboratory, Royal Manchester Children's Hospital, Pendlebury, Manchester M27 $1 H A$.}

REFERENCES

Bailev, C. C., and Komrower, G. M. (1974). Growth and skeletal maturation in congenital adrenal hyperplasia: review of 20 cases. Archives of Disease in Childhood, 49, 4.

Komrower, G. M., and Longson, D. (1961). The treatment of congenital adrenal hyperplasia with a depot preparation of prednisolone. Acta Endocrinologica, 36, 157. 